\title{
Comparative Analysis of Rainfall Trends in the Jinghe River Basin of China During 1959-2014
}

\author{
Xuerou Weng \\ Southwest University \\ Xunjian Long \\ Southwest University \\ Yan Ye (Dyye_china@126.com) \\ Southwest University \\ Yong Ye \\ Southwest University
}

\section{Research Article}

Keywords: rainfall trend, Mann-Kendall test, innovative trend analysis, innovative polygonal trend analysis, Jinghe Basin

Posted Date: November 3rd, 2021

DOI: https://doi.org/10.21203/rs.3.rs-870481/v1

License: (1) (1) This work is licensed under a Creative Commons Attribution 4.0 International License. Read Full License 


\section{Comparative Analysis of Rainfall Trends in the Jinghe River}

\section{Basin During 1959-2014}

3 Xuerou Weng ${ }^{1,2}$, Xunjian Long ${ }^{1}$, Yan Ye ${ }^{*_{1}}$ and Yong Ye ${ }^{1}$

$4 \quad{ }^{1}$ College of Resources and Environment, Southwest University, Chongqing, China

$5 \quad 2$ School of Geography and Planning, Sun Yat-sen University, Guangdong, China

6 * Corresponding Author: Yan, Ye College of Resources and Environment, Southwest University,

7 Tiansheng Road, Beibei, Chongqing, China. email: yye china@126.com

\section{Abstract}

9 Trend analysis is widely applied in hydrometeorological research. Considering that Innovative

10 Trend Analysis (ITA) and Innovative Polygonal Trend Analysis (IPTA) can detect small variations

11 on annual and smaller scale, rainfall trends at 14 hydrometeorological stations in the Jinghe River

12 Basin were analyzed by ITA, IPTA and Mann Kendall test (MK). The results showed that the rainfall trends are subsistent from 1959 to 2014. Comparing the results of ITA and MK on annual level, it was determined that trends are consistent, but only two stations passed the $90 \%$ significance test through MK, while all stations passed the significance test through ITA. Accordingly, the ITA method proved to be better than MK in detecting small changes in time series. Changes in high and low values, obtained by the ITA method, reflected flood and drought trends in the basin. In addition, IPTA is an improved ITA method that is suitable for a relatively short time span. Through the IPTA method for analyzing the monthly precipitation trends, the results showed that rainfall at 14 stations increased in January, February, March, June and December, and decreased significantly in September. Therefore, the methodology applied in this study can provide detailed recommendations for hydrometeorological research.

Keywords: rainfall trend; Mann-Kendall test; innovative trend analysis; innovative polygonal trend analysis; Jinghe Basin 


\section{INTRODUCTION}

Rainfall is a basic component of the global water cycle (Michaelides et al., 2009), and also one of the most important input variables in climate and hydrological research. For arid and semi-arid areas, the change in precipitation is directly related to the change of surface runoff and regional dry and wet conditions. According to the Fifth Assessment of the Intergovernmental Panel on Climate Change (CMIP5)(IPCC, 2014), the global mean surface temperature has increased $0.74{ }^{\circ} \mathrm{C}$ over the last century. The increase in global temperature is affecting and will continue to affect the water cycle, leading to changes in rainfall distribution(Chen et al., 2014; Zhang, Gao,\&Zhao, 2003). Previous research(Wen, Wu,\& Gao, 2017; Yang, Xia, Zhang,\& Hong, 2017) showed that rainfall in arid and semi-arid areas in western China, especially in the northwestern part, has been increasing in recent years. Accordingly, accurate estimation of the long-term rainfall trends has become an increasingly active research topic for effective regional management of water resources. Numerous methods are available for the trend analysis of hydrological and meteorological time series.

Nonparametric tests are an important part of the statistical analysis. In the past two decades, they have been widely used for the analysis of hydrometeorological time series. As the classic mathematical methods, Mann-Kendall (MK) test, Spearman's Rho (SR) test, regression analysis and wavelet analysis are widely recommended(Malik et al., 2020; Nourani, Baghanam, Adamowski,\& Gebremichael, 2013; Zarei,Moghimi,\& Mahmoudi, 2016).

However, the above-mentioned methods for trend analysis are pure statistical methods that do not allow the identification of trends in low, medium, and high values in a single calculation process. Recently, the innovative trend analysis (ITA) method proposed by Şen(Şen, 2017) was applied to detect trends in hydrological and meteorological parameters in different regions of the world(Chandole, Joshi\& Rana, 2019; Malik et al., 2020; Pour, Wahab, Shahid,\& Ismail, 2020). Although studies(Serinaldi, Chebana,\& Kilsby, 2020) have shown that the ITA method is influenced by sample size, distribution shape, and serial correlation, its advantages can be applied in different aspects. Kisi(Kisi \& Ozgur, 2015) found that the graphs obtained by the ITA method can better reveal the hidden trends in pan evaporation, compared to the MK and SR tests. Once the ITA method was proposed, it is not only applied in different aspects and regions, yet many scholars have improved the ITA method. Şen(Şen, 2017) further improved the ITA method and developed a calculation approach to derive a monotonic trend and a significance test, making it easier to obtain the trend behavior for different time series categories (low, medium, and high)(Güçlü, 2018). The new ITA visualization(Güçlü, 2020) shows clearly a number of data unlike the ITA applications. In addition, the change point on difference series is identified by universal Pettitt test, and then two subcategories are objectively defined as "high" and "low" values. The ITA-change boxes (ITA-CB) approach(Alashan, 2018) was introduced for numerical visualization of changes in time series on the ITA scatter graph. In 
this approach, as opposed to ITA that calculates changes as quantities, the changes are calculated in percent according to the mean, while lower range and upper range indicate the expected minimum and maximum change in a time series. In addition, Sen also proposed the Innovative Polygonal Trend Analysis (IPTA), which can not only identify small-scale time series (i.e., daily, weekly, monthly, etc.), but also determine the trend transitions between successive sections of two equal segments of the original hydro-meteorological time series, thus leading to the polygon trend.

Most studies have confirmed the general warming and humidification trend in Northwest China, which is based on the long-term meteorological data(Chen, Xie,\& Zhen, 2007; Guo et al., 2015; Zhang, Gu, Xin,\&Qi, 2016; Zhang et al., 2011; Zhang, Wang, Yu, Zhang,\&Tu, 2010). The Jinghe River, located in the southeast of the northwestern China, is one of the top ten river systems in the Yellow River basin. Due to the low vegetation cover, serious soil erosion, intense human activities and overload of water resource capacity, the ecological environment of the basin is very fragile and the degradation of natural resources also decreases the sustainability of the ecosystem(Buendia et al., 2016; Chen, Chang, Huang, Wang,\&Guo, 2014; Comino et al., 2017; Zhang, Gao,\&Zhao, 2003). In order to determine the variation characteristics of rainfall, the ITA method is applied to the annual rainfall in the Jinghe River Basin in order to effectively analyse the trends in precipitation, and also assess the possibilities of floods and droughts occurrences in the basin. Moreover, the monthly precipitation is examined using the IPTA graphical method, which provides a clear and intuitive understanding of precipitation changes in each month of the year, in order to actively and efficiently respond to soil erosion and vegetation degradation.

\section{DATA AND METHODS}

\subsection{Data}

The Jinghe River is the largest tributary of the Weihe River that is one of the important branches in Yellow River, and is located in the central part of the Loess Plateau in northwestern China. It originates in the Ningxia Hui Autonomous Region, and flows through the provinces of Gansu and Shaanxi. The main stream has a total length of $455 \mathrm{~km}$ and a drainage area of $45,400 \mathrm{~km}^{2}$. The basin has a typical temperate continental climate with average annual temperature from $8{ }^{\circ} \mathrm{C}$ to $13{ }^{\circ} \mathrm{C}$, and average annual precipitation from 350 to $600 \mathrm{~mm}$, mainly concentrated in summer and autumn (from May to September). The Jinghe River Basin and locations of rainfall and hydrological stations are shown in Fig. 1.

Hourly rainfall data from 14 meteorological stations in the basin and from eight meteorological stations around the basin are obtained. The time series selected for the analysis of precipitation trends in the Jinghe River Basin is from 1959 to 2014. These stations are almost evenly distributed within and around the Jinghe River Basin. 


\subsection{Methods}

\subsubsection{Mann-Kendall Test}

The Mann-Kendall test(Alashan, 2018; Kendall, 1990; Mann, 1945) was used in this study to explain sequences in precipitation trends and variability. The basic principle of this method is based on a comparison of the time series $(Z)$ in itself $\left(z_{1}, z_{2}, \cdots, z_{n}\right)$. If the examined data is larger (smaller) than the following, then $-1(+1)$ is added to the MK statistics $(S)$ (Eq. 1). Here, the variable $(i)$ varies from 1 to $n-1$, and the variable $(j)$ varies from $i+1$ to the data length $(n)$. This process is repeated for all data element and $S$ statistics are calculated and summed (Eq. 2).

$$
\begin{gathered}
\operatorname{Sgn}\left(z_{j}-z_{i}\right)=\left\{\begin{array}{ccc}
1 & \text { if } & z_{j}>z_{i} \\
0 & \text { if } & z_{j}=z_{i} \\
-1 & \text { if } & z_{j}<z_{i}
\end{array}\right\} \\
S=\sum_{i=1}^{n-1} \sum_{j=i+1}^{n} \operatorname{sgn}\left(z_{j}-z_{i}\right)
\end{gathered}
$$

It is assumed that $S$ has a normal probability distribution function (PDF) with zero mean and some variance (Eq. 3 and Eq. 4). Moreover, it is assumed that the examined time series initially have no trend as a null hypothesis $\left(H_{0}\right)$. If $H_{0}$ is rejected, the alternative hypothesis $\left(H_{l}\right)$ is valid, which means that there is a trend in the analyzed time series. Acceptance is done according to standardized test statistic $(z)$ and a certain significance level (Eq. 5). If calculated $z$ value $\left(z_{\text {cal }}\right)$ is bigger than the tabulated normal distribution value $\left(z_{t a b}\right)$ according to the significance level $\alpha$, then there is a significant trend in time series.

$$
E(S)=0
$$

$$
\operatorname{Var}(S)=\frac{n(n-1)(2 n+5)}{18}
$$

$$
z=\left\{\begin{array}{ccc}
\frac{S-1}{\operatorname{Var}(S)} & \text { if } & S>0 \\
0 & \text { if } & S=0 \\
\frac{S+1}{\operatorname{Var}(S)} & \text { if } & S<0
\end{array}\right\}
$$

\subsubsection{Innovative Trend Analysis Method}

The idea of innovative trend analysis method is defined as follows:

1) A recorded hydro-climatic time series data, $a_{1}, a_{2} \cdots a_{n}$, is divided into two groups with equal length:

$$
\left\{b_{1, \mathrm{n} / 2}\right\}=\left\{a_{1}, a_{2}, \cdots a_{n / 2}\right\} \text { and }\left\{b_{2, \mathrm{n} / 2}\right\}=\left\{a_{n / 2+1}, a_{n / 2+2}, \cdots a_{n}\right\}
$$

2) Both sub-series are sorted separately in ascending order: 


$$
\begin{aligned}
& \left\{S_{1}\right\}=\left\{\min \left(b_{1, n / 2}\right), \cdots b_{i}, \cdots, \max \left(b_{1, n / 2}\right)\right\}(1<i<n / 2) \\
& \left\{S_{2}\right\}=\left\{\min \left(b_{2, n / 2}\right), \cdots b_{j}, \cdots, \max \left(b_{2, n / 2}\right)\right\}(1<j<n / 2)
\end{aligned}
$$

3) The first sub-series $\left\{S_{1}\right\}$ are located on the x-axis, and the other sub-series $\left\{S_{2}\right\}$ are on the

y-axis (Fig. 2). The 1:1 $\left(45^{\circ}\right)$ straight-line represents no trend line. If scattered points fall above (below) the 1:1 line, the time series show a monotonous increase (decrease) trend. Otherwise, if scattered points show non-monotonic trend, the time series are classified into several clusters. As shown in Fig. 2, the classification of hydro-meteorological data into "low", "medium", and "high" groups can be achieved by dividing the domain of data variation into three intervals. This enables the detection of rainfall trends for different clusters with important implications: high rainfall indicating flooding and low rainfall indicating drought(Oztopal \& Sen, 2017). In this study, groups of low, medium, and high values are arranged according to expert view, or variation over time is considered in three strictly objective manners by considering the first half-time series $\left(S_{1}\right)$ mean $(\bar{S})$ and standard deviation $\left(S_{d}\right)$, which helps determine the range of low, medium, and high values as: $S_{1}<\bar{S}-S_{d}, \bar{S}-S_{d}<S_{1}<\bar{S}+S_{d}$, and $S_{1}>\bar{S}+S_{d}$.

4) Meanwhile, the trend slope is calculated according to the following equation:

$$
b=\frac{2\left(\bar{S}_{2}-\bar{S}_{1}\right)}{n}
$$

where, $\bar{S}_{1}$ and $\bar{S}_{2}$ are arithmetic averages of the first and the second halves of the dependent variable; and $n$ is the number of data.

5) The standard deviation of the sampling slope can be obtained according to the following equation:

$$
\sigma_{b}=\frac{2 \sqrt{2}}{n \sqrt{n}} \sigma \sqrt{1-\rho_{\bar{S}_{1} \bar{s}_{2}}}
$$

where, $\rho_{\bar{S}_{1} \bar{S}_{2}}$ implies the cross-correlation coefficient between the arithmetic averages of the ascendingly sorted two-halves; and $\sigma$ is the standard deviation of the whole series.

6) To test the significance of the trend slope value $(b)$, the null hypothesis $\left(H_{0}\right)$ implies that there is no significant trend if $b$ remains below the critical value $\left(b_{c r}\right)$. Otherwise, the alternative hypothesis $\left(H_{l}\right)$ is valid when $b>b_{c r}$. If at $\alpha$ percent significance level, the confidence limits of the standard normal PDF with zero mean and standard deviation is $b_{c r i}$, then the confidence limits $(C L)$ of the trend slope can be expressed with following equation:

$$
C L_{(1-\alpha)}=0 \pm b_{c r i} \sigma_{b}
$$

where, $b$ of each station falls outside the lower and upper $C L$, indicating the adoption of alternative hypotheses $\left(H_{a}\right)$ and indicating the existence of trends (Yes) as decisions.

\subsubsection{Innovative Polygon Trend Analysis}

IPTA (Şen et al., 2019) can be applied on several time scales (i.e., daily, monthly, annual, etc.). 
Considering a monthly hydro-meteorological time series as $x_{1}, x_{2}, \cdots x_{n}$, where $n$ is the number of years, this sequence can be arranged into a monthly matrix as:

$$
\left[\begin{array}{cccc}
x_{1,1} & x_{1,2} & \cdots & x_{1,12} \\
x_{2,1} & x_{2,2} & \cdots & x_{2,12} \\
\vdots & \vdots & \vdots & \vdots \\
x_{i, 1} & x_{i, 2} & \cdots & x_{i, 12} \\
\vdots & \vdots & \vdots & \vdots \\
x_{n, 1} & x_{n, 2} & \cdots & x_{n, 12}
\end{array}\right] \uparrow \text { Upper half (the first half, }(i=1,2, \cdots n / 2)
$$

This matrix can be considered in two halves as the upper (first) and lower (second) part, taking into account the indicator $i$ as $i=1,2, \cdots, n / 2$ and $i=n / 2+1, n / 2+2, \cdots n$, respectively. Subsequently, monthly averages from each half of the matrix column can be calculated as upper half $\left(\bar{x}_{u, 1}, \bar{x}_{u, 2}, \cdots \bar{x}_{u, 12}\right)$ and lower half $\left(\bar{x}_{l, 1}, \bar{x}_{l, 2}, \cdots \bar{x}_{l, 12}\right)$. Provided that the monthly averages are considered in two sets, then the IPTA version of the ITA template appears as in Fig. 3, where each month is shown as the vertex on the polygon. Consecutive monthly points are connected with straight lines, indicating the transition from one month to the next. The straight line provides a set of qualitative and quantitative information about trend. The scatter of monthly values on this graph depends on the effects of different hydro-meteorological phenomena, such as types, frequency and magnitude of storms, weather patterns, morphology, human settlement, vegetation, land use and land change.

\section{RESULTS}

\subsection{Annual Rainfall Trends}

Table 1 indicates the results of MK test for 14 stations according to confidence levels of $10 \%$ and 5\%. It is clear that majority of stations had positive trends (11 of 14 stations). However, only two stations (Changwu and Lintong) had significant positive trend (90\% confidence level), while there was no station with significant negative trend.

ITA results are given in Table 2 for each station according to confidence levels of $10 \%$ and $5 \%$. It is obvious, from the table that the trends are similar to those obtained using the MK method. The slope $(s)$ was dominated by significant positive values $(p<0.1$ or $p<0.05)$. Annual rainfall in Jingyang, Zhenyuan, and Fuping showed a decreasing trend, while other 11 stations showed increasing trend.

Comparison of Tables 1 and 2 clearly indicates that the ITA test gives slightly higher positive trends and lower negative trends than the MK method. According to the ITA method, Jingyang station, for example, shows a significant decreasing trend at a confidence level of 0.05 , while it has no significant trend according to MK method.

The ITA graphical results can be clearly seen from Fig. 4 since the scattering points of most stations fall above (below) the 1:1 line with high, medium, and low values in the Cartesian coordinate 
system. Generally speaking, there are more rainfall events that are classified as median, but fewer with high and low values. High rainfall is decreasing in Jingyang, Zhengning, Jingchuan, Lintai, while it is increasing in Changwu, Qingcheng. Tongchuan, Lintong and Fuping are relatively stable regarding high rainfall. In general, the change trend for other sites regarding high values is not obvious. Xunyi, Changwu, Huanxian, Zhenyuan, Ningxian, Jingchuan etc. have some points far from 1:1 line in the high value area. If they are above 1:1 line, it means that rainfall during 1987-2014, when extreme rainfall events occurred, has increased significantly compared to 1959-1986. If it is below, rainfall is significantly reduced. For the median region, most points at Jingyang, Zhenyuan and Fuping stations were below the 1:1 line, showing a decreasing trend. Tongchuan, Xunyi, Huating, Ningxian and Lintong are above the 1:1 line, showing an increasing trend. The trend for other sites is not significant. In the low value area, Tongchuan, Xunyi, Changwu, Ningxian, Zhengning, Qingcheng, Jingchuan and Lingtai stations showed an increasing trend, while Huanxian, Zhenyuan, Huating and Fuping stations were mostly on the 1:1 line, showing no obvious trend change. The lowest points at Jingyang, Lintong and Lingtai stations are below 1:1 and far away, which indicates that the severity of extreme drought events in the second half of the period is slower than in the previous half.

\subsection{Monthly Rainfall Trends}

The IPTA graphs are presented in Fig. 5 and they show the arithmetic mean changes of monthly rainfall in the first and second half of the sub-series at 14 stations in the Jinghe River Basin.

There is no regular and stable appearance of consecutive monthly points, as it is usually expected similar to the polygon in Fig. 3. Mean monthly rainfall is not stable, it shows non-systematic shifts from one month to another, and the rainfall does not show homogeneous and isotropic behavior. From the first sub-series (1959-1986) to the second sub-series (1987-2014), all 14 stations showed increasing trends in January, February, March, June and December, decreasing trend in September, while changes were different in the remaining months. Among the stations, the polygonal areas of Tongchuan and Zhenyuan are relatively small indicating that the monthly rainfall at these stations is relatively consistent with more stable occurrence of hydro-meteorological events.

In Jingyang, rainfall remained above the 1:1 straight-line during five months (January, February, March, June, and December), which indicates that there was more rainfall during these months in the recent investigated period (1987-2017) compared to the previous investigated period (1959-1987). The opposite situation is registered during remaining months with strong decreasing trends in September. Tongchuan, similar to Lingtai, shows increasing trends from January to March, and then decreasing trends until June. Substantial increasing trends are registered in June and July before the opposite occurs in August. From July to August there is a decreasing trend and then after October there are increasing trends until January. Stations Xunyi, Fuping, and Jingchuan have similar trends. For example, from January to March trends are above the 1:1 straight-line and then they change to the 
below part of the 1:1 straight-line. After April, transitions occur continuously from May to June, June to July, July to August, August to September and September to October, finally reaching January. Changwu and Ningxian show similar trends as Tongchuan, except for August. A fairly uniform polygon is obtained for HuanXian, with only four months (May, August, September, October) with decreasing trends and with transitions towards the decreasing area from May to April and from July to August. Transitions from increasing to decreasing trends happen from May to June and from decreasing to increasing happen from June to July in Zhenyuan. After July, decreasing trend remains for two months and then another significant transition occurs from September to October and remains for next six months, until the shift from increasing to decreasing area occurs between March and April. In Huating, only three months (April, July, and September) have a decreasing trend with transitions towards the increasing trend from April to May, July to August, and September to October. There are also transitions from increasing to decreasing trend from March to April, June to July, and August to September. In Lintong, except for April, September and October, other months are above the 1:1 line, showing an increasing trend. The monthly rainfall trends at Zhengning and Qingcheng are consistent with Changwu, except for July.

\section{DISCUSSION}

\subsection{Annual Rainfall Trends}

The ITA and MK methods were applied to analyze the trends in annual rainfall from 14 stations in the Jinghe River Basin during a 56-year period (1959-2014). The results showed that the MK test obtained only two significant trends, while the ITA test showed significant change at all 14 stations. In general, rainfall increased in the Jinghe River Basin from 1959 to 2014, which is consistent with the results of Yao et al.(2015) study concluding that the rainfall increased significantly in the Jinghe River Basin from 1957 to 2012.

Through the analysis of low rainfall values, it was determined that eight out of fourteen stations had increased amount of rainfall, while the other six stations did not have an obvious trend and many stations are below the 1:1 line at the lowest point of low rainfall. These results confirmed Zhang et al.(2016) analysis that used the Soil and Water Assessment Tool (SWAT) hydrological model to assess the spatial and temporal variation of drought characteristics in the basin. The study found that the basin has a "wet" trend, while drought has slowed in recent years. Despite this, the Jinghe River Basin is located in a drought prone area with severely restricted economic development. Accordingly, it is necessary to strengthen the resilience to drought, improve the region's emergency response capacities, and protect the water sources. The rainfall at each station is mostly concentrated in the median region. The more points in the median region, the less the possibility of extreme precipitation events in the region, and less drought and flood events occur, as at Huanxian Station. The trend of high rainfall 

in the central part of the Loess Plateau, which is one of the important sources of sediment for the Yellow River. However, soil erosion on the Loess Plateau is mainly caused by extreme precipitation events, such as heavy rainstorms(Buendia et al., 2016; Comino et al., 2017). The highest points of Xunyi, Changwu, Fuping and other stations in the high value area are all above the 1:1 line, and the distance is relatively large. It is necessary to pay special attention to the impacts of a significant increase in heavy rainfall events on regional soil erosion.

A number of studies(Chen et al., 2007; Guo et al., 2015; Zhang et al., 2011; Zhang et al., 2010) found that the rainfall changes were not significant in the basin, e.g., rainfall changes did not show obvious decreasing trend in the period 1950-2010, yet increasing trend was noticed in the 1960-2010 period. Accordingly, it can be noticed that the rainfall changes have a certain periodicity in the Jinghe River Basin, so the assessment of the certain periodic changes needs to be supported by additional rainfall data. However, development of meteorological stations is still relatively limited in the basin and thus it is difficult to obtain data.

Above all, it was found that the ITA method was more favorable in detecting small changes in rainfall time series, although this method, similar to the MK test, had limitations in sample size, distribution shape, and serial correlation(Serinaldi et al., 2020). Moreover, the analysis of high, medium, and low rainfall values can also provide a basis for the assessment of the possibilities for occurrence of extreme dry and wet events, such as droughts and floods(Dabanlı et al., 2016).

\subsection{Monthly Rainfall Trend}

The IPTA method was applied to analyze the monthly rainfall data from 14 stations in the Jinghe River Basin from 1959 to 2014, and the results showed increasing trend of rainfall at all stations in January, February, and December. It can be seen that the winter precipitation has an increasing trend in the Jinghe River Basin, which is consistent with the results of Chen et al(2007). The floods of Jinghe are mainly formed due to the summer rainstorms, with the precipitation mostly increasing from June to August at all stations. Therefore, in the period of frequent floods, the relevant departments should take measures for early flood control in order to deal with the possible increase in the floods flow. In addition, the Jinghe River Basin is located in the warm temperate-temperate, semi-humid-semi-arid transition zone, with fragile environment and weak water storage capacity. However, precipitation is concentrated in summer and is characterized by rainstorms and large runoff, which is not easy to control and preserve. Drought can occur in all seasons, with autumn being the season in which drought prevention is most needed(Chen et al., 2014). Precipitation significantly declines in September, which could be due to the influences of atmospheric circulations(Zhang et al., 2003). Therefore, September is the most significant month in autumn. In addition, the rainfall of most stations show a decreasing trend 
to October. Accordingly, it is also not difficult to predict that the impact of drought on crop growth could be more serious in the future.

Compared to ITA, MK and other methods, IPTA is the recommended method to analyze monthly rainfall and changes in monthly time series. Besides, the operational process is simpler, and the results of trend changes can be more clearly resolved by using the obtained figures.

292

\section{CONCLUSIONS}

The rainfall series are chaotic and random, and traditional methods could not obtain the samll temporal variations. To investigate the changing trend of rainfall, the ITA and MK tests were applied for annual variation, the IPTA was used for monthly trend detection, and the results are displayed as follows.

1) During 1959-2014, only $14 \%$ of stations had significant trends (90\%). However, the ITA method obtained that the rainfall increased significantly (95\%) at all stations (except Jingyang, Zhenyuan and Fuping) from 1959 to 2014. Compared to the MK test, ITA method is more conducive for the analysis of small changes in time series and provided an opportunity to distinguish the "low", "medium", and "high" rainfall values.

2) The monthly rainfall trends detected by the IPTA method at 14 stations in the Jinghe River Basin show that the rainfall increased in January, February, March, June, and December, but decreased in September. The IPTA method clearly and intuitively reflects the changes on a monthly time scale with the application of graphs. The results indicate that winter rainfall may increase and mitigate drought in this season.

Funding statement: this study was funded by Chongqing Municipal Training Program of Innovation and Entrepreneurship for Undergraduates (S202010635183) and Fundamental Research Funds for the Central Universities (XDJK2020C070).

Code availability: Not applicable

Ethical Approval: Not applicable

Consent to Participate: Not applicable

Consent to Publication: Not applicable

Author Contributions: Xunjian Long and Yan Ye conceived and designed the experiments; Xuerou Weng and Yong Ye performed the experiments; Xuerou Weng and Xunjian Long analyzed the data; Xuerou Weng, Xunjian Long and Yan Ye wrote the paper.

Data Availability Statement: Some or all data, models, or code used during the study were provided by a third party. Direct requests for these materials may be made to the provider as indicated in the Acknowledgments. The data that support the findings of this study are available in [China Meteorological Data Service Centre] at [www.data.cma.cn]. These data were derived from the following resources available in the public domain: [Hourly Data From Surface Meteorological Stations In China and http://data.cma.cn/en/?r=data/detail\&dataCode=A.0012.0001] 
ACKNOWLEDGMENTS: This research is supported by Chongqing Municipal Training Program of Innovation and Entrepreneurship for Undergraduates (S202010635183) and Fundamental Research Funds for the Central Universities (XDJK2020C070)

Conflicts of Interest: The authors declare no conflict of interest.

\section{References:}

Alashan. (2018). An improved version of innovative trend analyses. Arabian J. Geosci., 11(3), 50.

Buendia, C., Bussi, G., Tuset, J., Vericat, D., Sabater, S., Palau, A., \& Batalla, R. J. (2016). Effects of afforestation on runoff and sediment load in an upland Mediterranean catchment. Sci. Total Environ., 540

Chandole, V., Joshi, G. S., \& Rana, S. C. (2019). Spatio -temporal trend detection of hydro -meteorological parameters for climate change assessment in Lower Tapi river basin of Gujarat state, India. J. Atmos. Sol.-Terr. Phys., 195, 105130.

http://doi.org/https://doi.org/10.1016/j.jastp.2019.105130

Chen, C., Xie, G., \& Zhen, L. (2007). Characters of Precipitation Variation in Jinghe Watershed. Resour. Sci., (02), 172-177. http://doi.org/10.3321/j.issn:1007-7588.2007.02.026

Chen, Y., Chang, J., Huang, S., Wang, Y., \& Guo, A. (2014). Variation characteristics of drought in Weihe River Basin based on Palmer drought severity index. J. Nat. Disasters, 23(05), 29-37. http://doi.org/10.13577/j.jnd.2014.0504

Comino, J. R., Senciales, J. M., Ramos, M. C., Martínez-Casasnovas, J. A., Lasanta, T., Brevik, E. C., Ries, J. B., \& Sinoga, J. D. R. (2017). Understanding soil erosion processes in Mediterranean sloping vineyards (Montes de Málaga, Spain). Geoderma, 296

Dabanlı, İ., Şen, Z., Yeleğen, M. Ö., Şişman, E., Selek, B., \& Güçlü, Y. S. (2016). Trend Assessment by the Innovative-Şen Method. Water Resour. Manage., 30(14), 5193-5203. http://doi.org/10.1007/s11269-016-1478-4

Güçlü, Y. S. (2018). Multiple Şen-innovative trend analyses and partial Mann-Kendall test. J. Hydrol., 566, 685-704.

Güçlü, Y. S. (2020). Improved visualization for trend analysis by comparing with classical Mann-Kendall test and ITA. J. Hydrol., 584, 124674. http://doi.org/https://doi.org/10.1016/j.jhydrol.2020.124674

Guo, A., Huang, Q., Wang, Y., Li, Y., Chang, J., \& Mo, S. (2015). Detection of variations in precipitation-runoff relationship based on Archimedean Copula. J. Hydro. Eng., 34(6), 7-13. http://doi.org/10.1093/ndt/gfn256

IPCC. (2014). Climate change 2014: impacts, adaptation, and vulnerability. Contribution of Working Group II to the Fifth Assessment Report of the Intergovernmental Panel on Climate Change. Geneva, The Intergovernmental Panel on Climate Change,2014.

Kendall, M. G. (1990). Rank Correlation Methods. Br. J. Med. Psychol., 25(1), 86-91.

Kisi, \& Ozgur. (2015). An innovative method for trend analysis of monthly pan evaporations. J. Hydrol., 527, 1123-1129.

Malik, A., Kumar, A., Najah Ahmed, A., Ming Fai, C., Abdulmohsin Afan, H., Sefelnasr, A., Sherif, M., \& El-Shafie, A. (2020). Application of non-parametric approaches to identify trend in streamflow during 1976 - 2007 (Naula watershed). Alexandria Eng. J., 59(3), 1595-1606. http://doi.org/https://doi.org/10.1016/j.aej.2020.04.006

Mann, H. B. (1945). Nonparametric test against trend. ECONOMETRICA, 13(3), 245-259. 
Michaelides, S., Levizzani, V., Anagnostou, E., Bauer, P., Kasparis, T., \& Lane, J. (2009). Precipitation: Measurement, remote sensing, climatology and modeling. Atmos. Res., 94, 512-533. http://doi.org/10.1016/j.atmosres.2009.08.017

Nourani, V., Baghanam, A. H., Adamowski, J., \& Gebremichael, M. (2013). Using self-organizing maps and wavelet transforms for space - time pre-processing of satellite precipitation and runoff data in neural network based rainfall - runoff modeling. J. Hydrol, 476, 228-243. http://doi.org/https://doi.org/10.1016/j.jhydrol.2012.10.054

Oztopal, A., \& Sen, Z. (2017). Innovative Trend Methodology Applications to Precipitation Records in Turkey. Water Resour. Manage., 31(3), 727-737.

Pour, S. H., Wahab, A. K. A., Shahid, S., \& Ismail, Z. B. (2020). Changes in reference evapotranspiration and its driving factors in peninsular Malaysia. Atmos. Res., 246, 105096. http://doi.org/https://doi.org/10.1016/j.atmosres.2020.105096

Şen, Z. (2017). Innovative trend significance test and applications. Theor. Appl. Climatol., 127(3), 939-947. http://doi.org/10.1007/s00704-015-1681-x

Şen, Z., Şişman, E., \& Dabanli, I. (2019). Innovative Polygon Trend Analysis (IPTA) and Applications. J. Hydrol, 575

Serinaldi, F., Chebana, F., \& Kilsby, C. G. (2020). Dissecting innovative trend analysis. STOCH. ENV. RES. RISK A., 34(5), 733-754. http://doi.org/10.1007/s00477-020-01797-x

Wen, X., Wu, X., \& Gao, M. (2017). Spatiotemporal variability of temperature and precipitation in Gansu Province (Northwest China) during 1951 - 2015. Atmos. Res., 197, 132-149. http://doi.org/https://doi.org/10.1016/j.atmosres.2017.07.001

Yang, P., Xia, J., Zhang, Y., \& Hong, S. (2017). Temporal and spatial variations of precipitation in Northwest China during 1960 - 2013. Atmos. Res., 183, 283-295. http://doi.org/https://doi.org/10.1016/j.atmosres.2016.09.014

Yao, J., Zhao, Q., \& Liu, Z. (2015). Effect of climate variability and human activities on runoff in the Jinghe River Basin, Northwest China. J. Mt. Sci., 12(2), 358-367.

Zarei, A. R., Moghimi, M. M., \& Mahmoudi, M. R. (2016). Parametric and Non-Parametric Trend of Drought in Arid and Semi-Arid Regions Using RDI Index. Water Resour. Manage, 30(14), 1-22. http://doi.org/https://doi.org/10.1007/s11269-016-1501-9

Zhang, C., Gao, X., \& Zhao, H. (2003). Impact of global warming autumn precipitation in northwest China. J. Glaciol. and Geocry.(02), 157-164. http://doi.org/ 10.3969/j.issn.1000-0240.2003.02.007

Zhang, H., Gu, L., Xin, C., \& Qijun, Y. (2016). Investigation on the Spatial-temporal Variation of Drought Characteristics in Jinghe River Basin. J. North China University of Water Resources and Electric Power(Natural Science Edition), 37(03), 1-10. http://doi.org/10.3969/j.issn.1002-5634.2016.03.001

Zhang, S., Wang, Y., Yu, P., Zhang, H., Liu, G., \& Tu, X. (2011). Spatio-temporal Variance of Annaul Runoff in Jinghe River Basin of Northwest China in Past 50 Years and Its Main Causes. Sci. Geogr. Sin., 31(06), 721-727. http://doi.org/10.13249/j.cnki.sgs.2011.06.018

Zhang, S., Wang, Y., Yu, P., Zhang, H., \& Tu, X. (2010). Study for Separating the Impact of Precipitation Variation and Human Activities on Runoff Change of the Upper Reaches of Jing River. J. Soil Water Conserv., 24(04), 53-58. http://doi.org/10.13870/j.cnki.stbcxb.2010.04.001

\section{Figures \& Tables}

Fig. 1 Location and distribution of rainfall stations in the Jinghe River Basin 
Fig. 2 Illustration of the ITA method

Fig. 3 Illustration of the IPTA method

414 Fig. 4 ITA results for the annual rainfall at 14 stations in the Jinghe River Basin in the period 1959-2014

Fig. 5 IPTA results for monthly rainfall at 14 stations in the Jinghe River Basin for the period 1959-2014

Table 1. Results of the MK test for annual rainfall at 14 stations in the Jinghe River Basin for the period 1959-2014

Table 2. Results for Innovative Trend test (slopes) of annual rainfall at 14 stations in the Jinghe River Basin for the period 1959-2014 


\section{Figures}

\section{Figure 1}

Location and distribution of rainfall stations in the Jinghe River Basin

\section{Figure 2}

Illustration of the ITA method

\section{Figure 3}

Illustration of the IPTA method

\section{Figure 4}

ITA results for the annual rainfall at 14 stations in the Jinghe River Basin in the period 1959-2014

\section{Figure 5}

IPTA results for monthly rainfall at 14 stations in the Jinghe River Basin for the period 1959-2014

\section{Supplementary Files}

This is a list of supplementary files associated with this preprint. Click to download.

- Table1.xlsx

- Table2.xlsx 